\title{
Entre el cuerpo y la mística: interacción del judeo-cristianismo en dos cuentos de Alejandro Jodorowsky
}

\section{Resumen}

Luis Aránguiz Kahn Irarangu@uc.cl

Pontificia Universidad Católica de Chile Chile

El continente latinoamericano fue conquistado por los españoles en el siglo XVI. Ellos trajeron consigo la autoridad de la Iglesia Católica, y ésta impuso su voluntad en todo el territorio hasta el siglo XX. Sin embargo, junto con ella, también entró a Latinoamérica el judaísmo. Alejandro Jodorowsky es un chileno de origen judío cuya literatura inicial tiene rasgos compartidos con ambas tradiciones. Por lo tanto, este ensayo profundizará en la relación entre literatura, cultura y religión a través del análisis de dos de sus cuentos.

Palabras clave: Religión - Cultura - Mística - Cuerpo

\begin{abstract}
The Latin American continent was conquered by the Spanish in the sixteenth century. They brought the authority of the Catholic Church, and it imposed its will on the continent until the twentieth century. However, along with her, also entered Judaism Latin America. Alejandro Jodorowsky is a Chilean Jewish origin whose early literature has shared features with both traditions. Therefore, this study further on the relationship between literature, culture and religion through the analysis of two of his stories.
\end{abstract}

Keywords: Religion - Culture - Mystic - Body

Los cuentos "Zipelbrum" y "La muerte del Rebe" fueron escritos por Alejandro Jodorowsky (Chile, 1929) y reunidos en un libro de cuentos y fábulas titulado "El paso del ganso", publicado en la década del '50. En este ensayo nos hemos propuesto examinar cómo nuestro autor conjuga elementos comunes de la 
tradición religiosa occidental judeo-cristiana. El análisis se centrará en la noción que ambos cuentos comparten de la relación hombre-Dios, y también en cuanto a la naturaleza humana, la relación cuerpoespíritu. Pensamos que Jodorowsky crea una dialéctica entre ambas, amparado principalmente en la noción del hecho místico y religioso. Por lo tanto, el trabajo aquí expuesto, más que un examen meramente literario, pretende ser también cultural.

\section{El problema de la cultura}

Si hacemos un recorrido a través de la historia del ser humano, constataremos que existe un elemento que está siempre arraigado a la cultura. Nos referimos a la religión.

Religión. ¿Qué es? ¿De dónde procede? Cada disciplina de estudio podría ofrecernos una respuesta diferente. Aquí entenderemos la religión desde el punto de vista de Raimon Panikkar:

"Podríamos describir la religión como aquel proyecto que intenta religar el hombre a toda la realidad para liberarlo de su solipsismo estableciendo vínculos liberadores, que empiezan consigo mismo. Así podríamos afirmar que una de las funciones de la religión consiste precisamente en sanar la herida que la escisión entre alma y cuerpo ha creado en el ser humano" (Panikkar,1998, p. 15).

Esta explicación funcionalista de "religión" permite ampliar lo que entendemos de aquella. Religión no es un conjunto de ritos establecidos por un credo determinado, sino todo aquello que religa al hombre con la noción que tenga acerca de lo divino, aun cuando se trate de una noción particular o individual de lo trascendente.

La razón para escribir este ensayo es dar cuenta de cómo la religión ha quedado plasmada en la obra literaria de Alejandro Jodorowsky. En su obra encontramos alusiones a muchas otras creencias, y hasta podríamos hablar de un cierto sincretismo religioso al interior de su literatura. Pero Jodorowsky plantea algo más interesante. En su obra encontraremos alusión a su raíz genealógica y también a su raíz cultural occidental, que inevitablemente se refiere al cristianismo. 
Esta idea de la unión intelectual entre las raíces genealógicas y las raíces culturales puede ser comprendida en lo que llamaré la geneacultura. Este enlace indisoluble es el que permite que escritores como Jodorowsky lleven a cabo una literatura geneacultural, en la que confluye la influencia de la tradición familiar como la de la tradición cultural. En este caso particular encontramos un enlace formado por su tradición genealógica judía y por la tradición cultural judeo-cristiana del contexto en el que se formó. Este modelo podría ser aplicado a literaturas insertas en otros contextos culturales, en las cuales también sería posible encontrar geneacultura.

El filósofo francés posmoderno, Jean-Luc Nancy, ha señalado que "El cristianismo es inseparable de Occidente (...) y todo nuestro pensamiento es de un extremo al otro cristiano" (Nancy, 2000, p. 11). Este axioma se refiere a la imposibilidad de quitar la influencia cristiana al pensamiento occidental. Sin duda alguna, no es sólo cristiana. Robert Schlette examinó una teoría anterior similar, que planteaba que nuestra forma de pensar como occidentales es cristiana aun cuando no lo queramos así; esto es lo que se entiende por "cristianización estructural" del pensamiento. No obstante, Schlette dijo que esto podría ser una "hebraización estructural" debido a que la "(...) estructura a que nos hemos referido aquí (cristiana), aparece ya dibujada en el Antiguo Testamento y no sólo en el Nuevo, cuya manera de pensar es la del Antiguo" (Schlette,1969, p. 102). Por lo tanto, es posible pensar en que el pensamiento occidental está íntimamente ligado al marco de pensamiento que se instituye a partir de la religión, y específicamente al judeo-cristianismo.

Estos puntos de vista platean la imposibilidad de disociar cultura occidental y judeo-cristianismo. El modelo filosófico expuesto nos permite comprender el contexto en el que se desarrolla Jodorowsky. Su obra está emparentada -y en parte es fruto- de la cultura del autor. La relación entre religión y cultura en ambos cuentos será revisada luego de explicar algunos tópicos preliminares imprescindibles. 


\section{Sobre religión, cuerpo y espacio}

Para examinar los cuentos desde una óptica literaria nos serviremos de la teoría del "Arte de las distribuciones" propuesta por Michael Foucault incluida en su libro Vigilar y Castigar. En esta obra, desarrolla el concepto de disciplina, el cual puede ser relacionado directamente con la lógica ascética. A pesar de que Foucault diseña esta teoría pensando en cárceles, recintos militares y hospitales, utilizaremos sus planteamientos como estructura funcional en el análisis de la espacialidad de los cuentos. Su teoría de "El Arte de las distribuciones" se divide en los siguientes puntos: Clausura, División en Zonas, Emplazamientos Funcionales, Sistemas de Relaciones, los que serán explicados y utilizados en el desarrollo del análisis.

Para examinar el asunto específico de la religión y la mística del cuerpo utilizaremos la teoría de Raimon Panikkar en el ensayo "Religión y Cuerpo". Trata la relación entre cuerpo y espíritu en la siguiente definición: "Cuerpo sería lo opuesto a espíritu, siendo este último invisible y el cuerpo visible (...) el cuerpo es la apariencia, lo que aparece, el fenómeno" (Panikkar, 1998, p. 21). A partir de esto, elabora una teoría que consiste en exponer las diversas formas de relación existentes entre el cuerpo y el espíritu en el hecho religioso. Estas son: truncada, que se demuestra en desprecio y/o abandono del cuerpo; e instrumental, que se da de manera positiva o negativa.

Además, en el último capítulo retomaremos el problema de la mística en el judaísmo y en el cristianismo. Habrá que hacer un examen entre los dos cuentos, y entre las dos tradiciones religiosas. Para esto, hemos de utilizar dos textos fundamentales: el tema del judaísmo lo veremos desde la óptica de Gershom Scholem, estudioso judío; y el tema del cristianismo lo abordaremos desde la óptica del católico Raimon Panikkar en su libro De la mística.

Para comodidad del lector, las citas de los cuentos se distinguirán de las demás en que sólo estarán seguidas del número de página. 


\section{Zipelbrum}

Este cuento relata la historia de Octavio, un joven estudiante universitario aparentemente de la Edad Media. Planteaba que la voz no depende de las cuerdas vocales, pues solamente está sujeta a los músculos y, por lo tanto, debe ser liberada del cuerpo. Se fue de la ciudad universitaria y arrendó una pieza de pensión. No era muy conocido porque siempre pasaba encerrado, y su alimentación era precaria. Un día salió presuroso de su habitación a caminar porque se hizo una herida, y de ella brotó la voz, asunto que confirmó su teoría. Es entonces cuando cae ante la puerta del zapatero Maese Brumstein, quien lo hace entrar y lo reconoce como una extraña deidad llamada "Zipelbrum" a la cual esperaba ansioso con plegarias y cantos. Resulta de esto que el zapatero cuelga a Octavio de una pared, le azota y luego le abre el tórax; al hacer esto último, la voz sale de Octavio. Finalmente lo tira al patio... donde lo comen los perros.

En este breve resumen ya vislumbramos algunos elementos principales del cuento.

\section{II.I. El cuerpo y el espacio}

"Su cama se pobló de parásitos y tuvo que acostumbrarse a las privaciones: podía permanecer semanas masticando pan duro y bebiendo agua" (85).

"Zipelbrum" es un cuento que se construye a base de -y se desarrolla en- lugares cerrados. En primer lugar, tenemos al pensionista Octavio. Su relación con los co-habitantes era nula, pues "Como no se asomaba al corredor, llegaron a olvidarlo" (85). Esto implica que el personaje estaba usualmente encerrado. Se abstraía de los demás para trabajar en su teoría.

Este espacio determinado, con lindes claros, se caracteriza por su suciedad. La cita que encabeza este apartado muestra a un Octavio que se desenvuelve en un espacio sucio. $Y$ en segundo lugar, nos da a conocer sus privaciones alimenticias. Estos dos elementos, suciedad y privación alimenticia, dan cuenta de la relación entre Octavio y su apariencia: descuida el espacio en el que vive, y se descuida a sí mismo. Podemos suponer que no está interesado en lo que da a conocer de sí a otros, pues tampoco le interesa hacerse notar entre los pensionistas. 
La forma en que se muestran los espacios puede ser examinada en términos de Foucault como de Clausura, es decir, un espacio autónomo de los demás y cerrado sobre sí mismo (Foucault, 2009, p. 165). La habitación de Octavio es un espacio cerrado, diferente de las otras habitaciones por la propia presencia de su ocupante y la identidad que le transmite.

Además, a partir de estos antecedentes, Octavio desarrollaría lo que Panikkar consideró como una relación truncada entre cuerpo y religión. Lo llamativo de esta relación es que se produce simultáneamente en las dos variantes que plantea nuestro autor.

Octavio piensa que debe liberar la voz del cuerpo, y es por ello que se desprecia a sí mismo. En este sentido, el cuerpo es un obstáculo para el cumplimiento de su tesis. Por eso se alegra cuando, al hacerse una herida, la voz emana de su carne. No obstante, a pesar de este deseo de eliminar el obstáculo del cuerpo, Octavio en ningún instante del cuento se auto-lacera. En palabras de Panikkar, Octavio tiene una relación de desprecio con su cuerpo pues éste es: “(...) un obstáculo para la salvación del hombre (...) Hay que liberarse de su dominio sobre nosotros (...) La mortificación corporal es necesaria para la vida espiritual (...) El 'asceta' desprecia su cuerpo” (Panikkar, 1998, p. 31).

Sin embargo, existe una dialéctica: él se desprecia en el intelecto, pero no en la práctica. Este desprecio no se manifiesta físicamente, sino que se manifiesta como un abandono de su apariencia, y es por esto que se conjugan ambos modos de relación. El abandono de su cuerpo es la forma en que se da conocer su desprecio por el cuerpo, y este mismo abandono se reproduce en el espacio habitado. En este sentido, el espacio equivale a una prolongación del estado de ánimo del personaje. Lo que llamaremos espacio habitado, se conjuga con la personalidad del personaje que lo habita.

La habitación de Octavio tiene una funcionalidad definida claramente: es ahí donde realiza sus experimentos fonéticos. Por lo tanto, su habitación no es un espacio muerto, sino un espacio que le presta utilidad. Este detalle nos permite retomar la teoría de Foucault y encontramos que también está presente el tercer punto de "El Arte de las distribuciones". Nuestro autor habla de un Emplazamiento 
funcional, es decir, que el espacio inútil se transforma en el lugar que el sujeto habita para desarrollarse (Foucault, 2009, p. 167).

\section{II.II. El cuerpo y la religión}

Esta relación configurada por el cuerpo y el espacio es básica para comprender la transformación que se da en el espacio de Maese Brumstein. La aparición de Octavio en su puerta es un hecho que transgrede las normas espaciales configuradas hasta ese momento. Brumstein era un zapatero alcohólico que se dedicaba a elevar rezos a Zipelbrum, (una extraña deidad creada por él mismo); al ver a Octavio, se encuentra con quien podría ser su dios. En este personaje encontramos una clara relación de abandono hacia su cuerpo debido a su alcoholismo.

El episodio gira en torno a lo que sucede con Octavio, puesto que su cuerpo demacrado parecido a la madera, es muy similar al "(...) muñeco de madera con voz humana que un día iba a llegar para darle felicidad (a Brumstein)" (86). Maese lo introdujo a su habitación, lo colgó de la pared como un crucifijo y le prendió tres velas. Este cuadro muestra la irrupción de lo que Brumstein consideraba divino, en su vida cotidiana de ilusión y desconsuelo. El elemento que le sirvió para reconocer a Zipulbrum en Octavio es el medio de la religiosidad por excelencia: su cuerpo.

El cuento relata que una de las características principales de Zipelbrum es que está hecho de madera, y la piel de Octavio en el momento en que llegó a la puerta de Maese Brumstein parecía madera. El cuerpo de Octavio funciona como el mediador cognitivo entre la realidad de Octavio y la realidad de Maese Brumstein. Es interesante notar la relación que hay entre el cuerpo, como si fuera de madera, y el hecho de ser colgado a la pared. Esto nos remite directamente al símbolo religioso. El cuerpo de Octavio cumple la función que destaca Panikkar en su noción del cuerpo como fenómeno y como apariencia. El cuerpo de Octavio es la apariencia de Zipelbrum a los ojos de Brumstein, aunque ni el mismo Octavio lo sepa.

Maese cuelga el cuerpo de Octavio a la pared y se postra delante de su él como si fuera un ídolo. Se produce una interacción entre altura/bajeza y específicamente ídolo/adorador. El cuerpo de Octavio es 
puesto en la altura como un objeto de reverencia ante el cual Maese se postra de rodillas. El espacio habitado por Maese Brumstein está ligado a la noción foucaultiana de Sistema de Relaciones, que postula que dentro de un espacio cada cuerpo se distingue de otro según el lugar que ocupe: "Su unidad (...) es el rango: el lugar que se ocupa en una clasificación (...) individualiza los cuerpos mediante una localización que no los implanta, pero los distribuye y los hace circular en un sistema de relaciones" (2009, p. 169). El Sistema de Relación es asimétrico dado que uno reconoce la superioridad del otro. Esta imagen se asemeja a la de un feligrés arrodillado frente a un crucifijo con el cuerpo llagado de Jesús. Un nexo importante en esta relación se da cuando Maese comienza a golpear a Octavio colgado, hasta que le hace heridas profundas. Ese episodio remite al pasaje evangélico en que Jesús es azotado (Mc. 14,65). Luego Brumstein rompe con un cuchillo el tórax de Octavio (88), lo cual también remite al episodio en que es atravesado por una lanza (Jn. 19,34). El juego simbólico con lo religioso se hace patente, y nuevamente, el medio es el cuerpo. Así, lo que permite establecer el nexo entre el hecho religioso y un cuento aparentemente distante de la religión cristiana es la conformación de los elementos corpóreos que Jodorowsky dispuso.

\section{La muerte del Rebe}

Este cuento relata la historia de Anán y su hijo Todros. El padre, ansioso de buscar la pureza, se embarca junto a su hijo en la empresa de una vida ascética y libre del placer. Comienzan a vivir en una cueva y se alimentan de frutos naturales y de lo que las personas en un pueblo cercano les pueden dar. Sin embargo, un día el padre de Todros sale a buscar comida mientras él iniciaba un viaje espiritual. Dos osos entran a la cueva y devoran su cuerpo. Al descubrir esto, Anán se enfurece y sale a buscar a los osos para matarlos. Olvida sus principios de fe y tiene una relación sexual con una mujer que estaba emparejada con un oso al cual mata.

\section{III.I. Ascesis}

Este es el primer elemento que podemos tomar del cuento: 
"El ascetismo es el único medio que tenemos de conservarnos puros. Tú y yo, hijo mío, renunciaremos al placer. $Y$ desde entonces comenzamos a usar vestiduras negras, a comer solo dos panes de cebada por día, uno en la mañana, el otro al atardecer; a rechazar la sal y el azúcar; a dormir en los suelos más duros" (114).

Es posible señalar que a partir de este hecho se suceden todos los acontecimientos posteriores. Por lo tanto, consideraremos como principal elemento del cuento a la mentalidad religiosa ascética.

El comportamiento descrito en la cita anterior puede ser clasificado dentro de lo que Panikkar llama relación instrumental, es decir, que el cuerpo es considerado como una herramienta para lograr un fin. Esta noción utilitaria se produce de modo negativo o positivo. Según la descripción del cuento, estamos frente a un caso de relación instrumental negativa en la que el cuerpo es un impedimento. Siendo éste "La sede de nuestros instintos bajos hay que someterle ascéticamente y tenerlo a raya. La mortificación del cuerpo y el control de los sentidos pertenece a una ascesis tradicional en todas las latitudes. (...) la razón debe dictar, dominar y utilizar el cuerpo" (Panikkar, 1998, p. 32).

Cuidar bien el cuerpo y evitar todo placer permite el perfeccionamiento interior que ayudará al asceta a lograr una mejor conexión con lo divino. A raíz de esto es que ambos personajes limitarán su dieta a un par de panes y rechazar todo aquello que pueda darle sabor (placer) a la comida, como son el azúcar y la sal. No obstante, con el paso del tiempo se dará una relación truncada de abandono, puesto que a Anán se le caen los dientes (114), y la muerte de Todros se deberá precisamente a que su cuerpo quedó en un abandono total.

\section{III.II. Espacio}

Comenzaron a vivir en una gruta (115), y dormían en suelos duros. En términos de espacialidad, la gruta se caracteriza como un espacio rocoso y lúgubre. Este espacio habitado se asemeja a una habitación oscura. En este sentido, la gruta cumple con la totalidad de los puntos de "El Arte de las distribuciones" según Foucault. En primer lugar, es un espacio de clausura porque está separado de otros y es cerrado en sí mismo. En segundo lugar, está dividido en zonas puesto que ahí ellos comen, duermen y también estudian la Torá. En palabra del autor: "A cada individuo, su lugar, y en cada emplazamiento, un 
individuo. (...) la disciplina organiza un espacio analítico" (Foucault, 2009, p. 166). En tercer lugar, es un espacio funcional en el cual ambos personajes se pueden desarrollar en diferentes actividades, es decir, es utilizable; y por último, existe en él un sistema de relación marcado por la diferencia padre-hijo.

La configuración de este esquema nos permite señalar la gruta como un espacio de disciplina muy adecuado para los fines que ambos se proponen. Por otra parte, los aludidos sólo salen de su gruta para buscar alimento; esto implica que el recinto es habitable pero no proporciona los requerimientos vitales para sobrevivir.

III.III. Cuerpo llagado

Ahora bien, todo esto comienza y concluye en la ascesis. Los dos personajes se entregarán por completo a su meditación: "Hijo mío, tenemos que decir a las necesidades del alma y del cuerpo: este no soy yo" (115). La perspectiva de su ascetismo es una negación total, en cuerpo y en alma.

Este pasaje trasluce una dialéctica entre lo efímero y lo eterno. Para alcanzar esto último, es necesario someter al cuerpo a una disciplina totalizadora.

El cuerpo, por lo tanto, es abandonado y se prescinde de él a tal punto que: “(...) en el calor agobiante del verano, en el frio despiadado del invierno, con la piel tan pegada a los huesos que parecíamos momias, seguimos meditando" (116). Nos encontramos con un cuerpo seco, descuidado y abandonado. Mientras Anán salía a buscar frutos, Todros se quedó meditando y alcanzó un estado de meditación en el que salió de su cuerpo. Anán se demoró algunos días y, “(...) cuando llegó a nuestra gruta, encontró unos pocos huesos mordisqueados y un trozo sanguinolento de cuero cabelludo: los restos de mi cuerpo. Por las huellas se dio cuenta de que eran dos osos los que me habían devorado" (118).

La dicotomía entre cuerpo y alma se acentúa cuando el cuerpo de Todros es devorado, pero a la vez su alma está en un estado de profunda conciencia espiritual que le permite observar todo. Esto queda manifestado en el hecho de que es él quien narra su propia historia y relata cómo vio todo lo que sucedía. 
El cuerpo carece de finalidad, es sólo un elemento útil que al cumplir su función deja de servir, puesto que Todros ya ha logrado su ansiado deseo de conocer el ámbito espiritual.

\section{Relación entre "Zipelbrum" y "La muerte del Rebe"}

Ya hemos realizado un examen de ambos cuentos. Ahora, por lo tanto, vamos a establecer los puntos de relación en sus dos ejes: el cuerpo y el espacio.

\section{IV.I. El cuerpo}

En ambos cuentos es posible observar que el cuerpo funciona como un medio de lo religioso. Esto es, que es visto como un elemento que permite establecer conexión con lo divino. Tanto Octavio como Anán y Todros están conscientes que para lograr sus fines, deben el uno apreciar el tiempo y la investigación por sobre el cuidado de su cuerpo, y los otros abstenerse de todo lo placentero para agradar a Dios.

En continuidad con lo anterior, el cuerpo es una herramienta ascética. En ambos relatos encontramos elementos comunes a este respecto: piel estirada y seca. En el caso de "Zipelbrum" Octavio tiene la piel como madera. Nótese aquí el elemento del color. La madera es café, y denota sequedad. La dicotomía cuerpo/espíritu podría darse en términos de tronco/savia, y específicamente cuerpo/voz. Luego, cuando Maese Brumstein hunde el cuchillo en el cuerpo de Octavio, es sugerente pensar en un hombre con un hacha abriendo un tronco; del árbol sale la savia, como la voz sale del cuerpo de Octavio.

Por otra parte, en "La muerte del Rebe" la dicotomía cuerpo/espíritu es superada, ya que Todros logra salir satisfactoriamente del cuerpo. A posteriori, existe una noción del cuerpo como una cáscara o envoltorio que transporta algo que debe ser liberado.

Otro elemento común mencionado es el de las comidas frugales. Los personajes involucrados en ambos relatos tienen muy poco trato con los alimentos.

Por último, todo lo que se ha mencionado concluye con el deseo fundamental de los personajes. El primero, liberar la voz; los otros dos, búsqueda de encuentro con el Innominable. Sin los elementos 
anteriormente mencionados, nos sería dificultoso descubrir el desenlace que, como se ha dicho, está íntimamente ligado a la mística.

\section{IV.II. El espacio}

En cuanto al espacio, hay tres elementos que se pueden destacar. En primer lugar, en sí mismo es un modo de disciplina. En ambos relatos los personajes requieren desarrollarse en lugares que les permitan ejercer su disciplina libremente. Preferiblemente, los lugares deben ser solitarios. En el caso de Octavio vemos que su espacio está inserto en una pensión, no obstante cuando se encierra tras la puerta, ese espacio es propio y al interior del mismo la disciplina investigativa se puede desarrollar sin dificultad. En el caso de Anán y Todros, la cueva es un lugar solitario que les permite desarrollar su disciplina ascética libremente.

En segundo lugar, el espacio asume una condición de limitación. Fuera de sus espacios, los personajes no pueden llevar a cabo su disciplina. Octavio tenía todos los implementos de estudio en su pieza. Anán y Todros se desarrollan al interior de la caverna y solamente salen por razones justificadas de supervivencia: cuando necesitan alimento.

En tercer lugar, los espacios se asumen retirados de la aglomeración. Esta condición es indispensable en ambos relatos. En "La muerte del Rebe" vemos que padre e hijo se apartan del pueblo y de la familia, y bajan a la población únicamente cuando tienen necesidad de alimento: "Bajábamos al pueblo a pedigüeñar cantando, una hora por día" (115). La ascesis requiere soledad que permita desarrollar las actividades espirituales. Fijémonos en lo importante que es el elemento del silencio. Luego, Octavio en "Zipelbrum" se margina de sus compañeros de pensión, y se abstrae de los sitios en que podría producirse aglomeración, como el comedor (85). 


\section{Sobre el elemento místico religioso judío-cristiano}

Dijimos al inicio que en ambos cuentos existe la conjunción cultural-religiosa de judaísmo y cristianismo. Ya hemos señalado rápidamente algunas relaciones que ahora intentaremos profundizar. Sin embargo, es necesario especificar que debido a la amplia extensión del tema, nos limitaremos a revisar la relación entre el cuento y la mística, y no al revés. Los conceptos serán precisados a medida que avancemos en el examen.

Panikkar define la mística como "aquella que nos permite gozar plenamente de la vida" (Panikkar, 2005, p. 25), y lo especifica señalando que "la experiencia de la vida es la experiencia del misterio, es la conciencia de que se está experimentando algo que no se puede pensar".

Vamos ahora a las diferenciaciones religiosas. La mística del judaísmo se caracteriza por la conjunción de tres elementos: relación Dios/creación, estudio de la Torá y Éxtasis.

En el caso del cristianismo, también se distinguen tres elementos: Encarnación (cuerpo, sangre), Cruz (sacrificio) y Resurrección (espiritualidad).

\section{V.I El cristianismo en Zipelbrum}

Una primera consideración es que el concepto de Cristo Salvador viene de la tradición mesiánica judía. En ese sentido, "Zipelbrum" es un cuento altamente judeo-cristiano y es un reflejo fiel de la interacción de tradiciones religiosas que he llamado geneacultura. Se utilizará la clave mística para interpretar dos puntos: el rol de la voz y el sujeto Octavio/Zipelbrum.

El tema de la voz puede ser interpretado desde una clave mística. Como ya hemos mencionado anteriormente, Octavio es un muchacho que postula la siguiente teoría:

"La voz no surge de las cuerdas vocales ni del aire que las remece. Existe sin que nadie la produzca. Sólo que está prisionera en los músculos de la garganta y depende de la voluntad... quiero liberarla. Hacer que salga por cualquier parte del cuerpo: por un ojo, por una mano. Conseguido esto, independizarla de mi voluntad. Entonces sonará cuando y por donde ella quiera. Yo la oiré" (85). 
De esta lectura rescatamos inmediatamente los siguientes conceptos sobre la voz: depende de la voluntad de quien la porta, es de origen desconocido, está prisionera, y debe ser liberada.

Coincidentemente, estas categorías están muy apegadas a las que veremos ahora con Panikkar. Entendemos la voz como el modo de comunicación sonoro por excelencia. La voz es sonido. Desde esta óptica, Octavio querría liberar su sonido, que está bajo su voluntad. Este es su fin. Él asume que la voz no tiene origen. Panikkar nos dice: “(...) el místico aspira a este Principio de la Palabra. Este principio "anterior" a la Palabra (que era en el Principio), pero no separable de ella, es el Silencio" (Panikkar, 2005, p. 43).

Octavio, al liberar la voz, queda en el silencio, un silencio que está íntimamente ligado a su muerte. La única forma de hacer que la voz fuera libre totalmente de él (de su voluntad) era el fin de su existencia. Octavio, aspirante al principio del silencio, pagó el deseo aquél con su vida.

Paradojalmente, "(...) la mística, bien entendida, es el reino de la libertad: libera al hombre tanto de sus condicionantes trascendentes como inmanentes" (Panikkar, 2005, p. 42), es decir, que el hombre alcanza una libertad espiritual plena. En el caso de nuestro cuento, estamos seguros de que la voz al fin es libre: “(...) abandonó el cadáver de su antiguo amo, recorrió el cuarto para después salir por la ventana y perderse hacia lo lejos" (88). De lo que no estamos seguros es si Octavio fue libre realmente. En términos dicotómicos, podríamos decir que la verdadera mística estaba en la voz, y no en Octavio.

Por otra parte, este cuento presenta una conexión con la mística cristiana en la persona misma de Zipelbrum-Octavio, de acuerdo a su interacción con Maese Brumstein. El concepto de encarnación en términos místicos significa que "La experiencia de Jesucristo nos descubre que existe un Hombre real que es a la vez Cuerpo (Materia) como yo soy, y divino (Espíritu), como yo aspiro a ser (...) no sólo es la divinización de un hombre (y con ello de todo hombre) sino también la humanización de Dios" (Panikkar, 2009, p. 247). Brumstein cree firmemente que Octavio es Zipelbrum, y lo reconoce como superior a penas le ve, “(...) es el muñeco de madera con voz humana que un día iba a llegar para darle felicidad" (86). Octavio, un joven común, es la encarnación de Zipelbrum. No es que Brumstein sea místico, es que 
en Octavio se cumple la aspiración de aquél. De hecho, la relación se invierte rápidamente. Ya que Zipelbrum/Octavio no puede dar respuesta a los problemas de Maese, éste comienza a golpearlo y azotarlo, hasta matarlo. Las categorías se invierten: el que era dios, es eliminado por aquél que lo reverenciaba, es decir, Brumstein tomó el poder y mató a su dios. Este es un nexo típicamente cristiano: Jesús, Dios, es negado por los hombres que supuestamente creían en él -a los que quería hacer el bieny luego es asesinado, solo. El poderoso se vuelve débil, y el débil poderoso.

La Cruz, por otra parte, es la “(...) experiencia de mi contingencia: que no me sostengo a mí mismo sino que me apoyo en lo Infinito (divino), aunque sea en un solo punto tangencial (contingencia). En esta experiencia descubro que no estoy solo" (Panikkar, 2005, p. 249). Nos preguntamos ¿qué pedía Maese Brumstein de Zipelbrum? Él pregunta: “- ¿qué felicidad esperas de mi? - “ique me paguen las deudas!” La felicidad de Maese Brumstein se reduce a la holgura económica debido a la pobreza de su presente, es decir, a su contingencia. Además, a esto agregamos el gesto mencionado en otra parte del trabajo: Octavio parecía de madera, y fue colgado en la pared, como un crucifijo cristiano. Octavio es un amuleto.

En cuanto a la Resurrección, se da un proceso inverso. Panikkar explica que "(...) si mi vida es un sacrificio, en su sentido real y tradicional, (Cristo) muriendo resucitó y resucitando restauró la calidad de vida" (Panikkar, 2005, p. 251). Zipelbrum/Octavio fue asesinado por su adorador, pero no resucitó. Esto implica que Maese Brumstein no accedió a la calidad de vida que esperaba, más bien seguiría siendo el borracho zapatero al que todos le debían dinero. Quizá sea esto lo que determinó la humanidad (y no la deidad) de Octavio. Es, por demás, el conflicto acerca de la persona histórica de Jesús.

\section{V.II El judaísmo en "La muerte del Rebe"}

En este relato la mística es fundamentalmente judía. Desde el inicio del cuento se evidencia esta tónica. Analizaremos el cuento desde los tres puntos fundamentales de la mística judía mencionados arriba.

La mística de Anán y Todros se formula como un intento del hombre por hallar los secretos del Innominable, se busca una relación entre Dios y la creación. Gershom Scholem explica que "En-sof es la perfección absoluta (...) No se revela a sí mismo de manera que resulte posible conocer su naturaleza, y 
no es accesible ni siquiera a los pensamientos más íntimos del contemplativo. Sólo través de la naturaleza finita de cada cosa que existe, a través de la existencia efectiva de la propia creación, se puede deducir la existencia de En-sof como la primera causa finita" (Scholem,1994, p. 115). Ambos están encerrados en la naturaleza humana, y la naturaleza externa es el espacio de contemplación, para entrar contacto con En-sof ( $\sin$ fin), que es Dios.

Además, ambos judíos estudiaban arduamente la Torá. Dejaban una hora para mendigar, tres para dormir, y por lo tanto veinte para estudiar: “(...) el resto del tiempo lo ocupábamos en leer la Torá, reservando tres escasas horas para dormir. Mi padre estaba seguro de que una frase de los Números encerraba el secreto de la vida eterna" (115). Este Estudio de la Torá se debe a que el judío (según Scholem) cree que "Dios se revela a sí mismo en ella (Torá) como Él es más que como un medio de comunicación en el sentido humano limitado" (Scholem,1994, p. 206). Es decir, la Torá es un medio de Dios para darse a conocer al hombre. Si el hombre quiere conocerle, dispone de la Torá para comenzar.

Por último, un elemento bastante marcado en el cuento es el Éxtasis en que entra Todros. Su padre le insiste que debe meditar, incluso durante el sueño. En este incesante meditar Todros dice: “(...) inmovilizando mi cuerpo esquelético, ignorando el frio, concentré mi espíritu en el camino estrecho. No podía ir ni a derecha ni a izquierda ni retroceder. Yo era el que pensaba, pero ¿Quién me pensaba? Yo era la conciencia, luego la conciencia de la conciencia" (116-17).

Esto se condice claramente con la afirmación de Scholem: “(...) la vía mística incluía esencialmente el ascenso del alma hasta un estado de rapto extático por un proceso de concentración del pensamiento y meditación" (1994, p. 214). Ahí se resuelve por qué el Anán le insistía tanto en la meditación.

\section{Conclusiones abiertas}

Jodorowsky representa un problema cultural latinoamericano. Es la imagen del problema de las religiones dominantes y las dominadas; pero también es la imagen de la enorme intercalación de religiones. La 
misma mezcla de elementos religiosos que emplea en sus cuentos se ve a diario en las religiosidades populares.

El problema cultural judeo-cristiano ha quedado al descubierto. La genealogía se ha mezclado con la cultura. "Zipelbrum" es una mezcla de elementos de ambas tradiciones y debe ser profundizado. "La muerte del Rebe", en cambio, es notoriamente judía. El fenómeno de la geneacultura está presente de manera explícita en la obra de nuestro autor.

El posible nexo fenoménico entre ambas tradiciones es el cuerpo. Es vehículo de la religiosidad, de todas las religiosidades. Quizá examinando cómo las distintas religiones se desarrollan a través de aquel, podrían hallarse diversos puntos de encuentro. Los cuentos muestran que el espacio habitado puede ser una señal de nuestro comportamiento corporal, y por lo tanto, de nuestro modo de pensar.

Hemos dado cuenta de la heterogeneidad de la obra de un autor que nos desconcierta, pero que nos revela cómo la literatura sigue siendo el medio de interacción cultural por excelencia. El camino queda abierto para seguir estudiando la geneacultura en la literatura judía cristiana de América Latina. 


\section{Bibliografía}

La Santa Biblia. Ed. castellana (Versión de Casiodoro de Reina y Cipriano de Valera). Revisión de 1960. Sociedades Bíblicas Unidas.

FOUCAULT, Michael. Vigilar y Castigar. Ed. castellana (Traducción de Aurelio Garzón del Camino). México DF, Siglo Veintiuno. 2009. 314p.

JODOROWSKY, Alejandro. "Zipelbrum". El paso del ganso. México DF, Mondadori. 2001. 156p.

JODOROWSKY, Alejandro. "La muerte del Rebe". El paso del ganso. México DF, Mondadori. 2001. $156 \mathrm{p}$.

NANCY, Jean-Luc. "La desconstrucción del cristianismo". Ed. Castellana (trads. Javier de la Higuera y José Carlos Bernal Pastor). Revista de Filosofía Año 37, (112): 7-28, 2000.

PANIKKAR, Raimon. De la Mística: experiencia plena de la vida. Barcelona, Herder. 2005. 302p.

PANIKKAR, Raimon. "Religión y cuerpo". EN: BOUSO, R. Y RODRIGUEZ, J. (Eds.) Estética y religión: el discurso del cuerpo y los sentidos. España, Er, 1998,11-48.

SCHOLEM, Gershom. Desarrollo histórico e ideas básicas de la Cábala. Ed. Castellana (traducción de J.S.B.). Barcelona, Riopiedras. 1994.

SCHLETTE, Robert. Cristianos y no cristianos. Ed. castellana (traducción de Eloy Revueltas Cruz). Barcelona, Herder. 1969. 